\title{
Physico-chemical, Antioxidant and Sensory Attributes of Ginger (Zingiber officinale) Enriched Jaggery of Different Sugarcane Varieties
}

\author{
M. A. Harish Nayaka - C. Vinutha • \\ S. Sudarshan • M. P. Manohar
}

Received: 9 October 2013/ Accepted: 9 June 2014/Published online: 7 August 2014

(c) Society for Sugar Research \& Promotion 2014

\begin{abstract}
Zingiber officinale enriched jaggery of three sugarcane varieties (Co 86032, Co 419 and Co 62175) at $0.05,0.1$ and $0.2 \% \mathrm{Z}$. officinale concentrations were evaluated for physico-chemical properties, 1,1-diphenyl-2picrylhydrazyl (DPPH) radical scavenging and reducing power ability, in addition to sensory evaluation by quantitative descriptive analysis method. Physico-chemical analysis of $Z$. officinale enriched jaggery revealed no significant difference between test and control except for total phenolics, tannins and flavonoids that indicated a dose dependent increase for all the varieties. A positive correlation $(\mathrm{r}=0.922,0.883$ and 0.881$)$ was observed between total phenolics and antioxidant activity of $Z$. officinale enriched jaggery for all the test varieties. Results of DPPH radical scavenging ability and reducing power potential of Z. officinale enriched jaggery showed an increased antioxidant activity. An $\mathrm{EC}_{50}$ of $3.098,3.076$ and $3.038 \mathrm{mg} /$ $\mathrm{mL}$ was observed in $0.2 \% \mathrm{Z}$. officinale enriched jaggery prepared from Co 86032, Co419 and Co 62175, respectively. Sensory evaluation of $Z$. officinale enriched jaggery for different attributes indicated significant $(P>0.05)$ difference between control and enriched jaggery of different sugarcane varieties for color, texture, hardness and taste.
\end{abstract}

Keywords Jaggery - Zingiber officinale .

Antioxidant activity $\cdot$ Sensory evaluation

M. A. Harish Nayaka $(\bowtie) \cdot$ C. Vinutha $\cdot$ S. Sudarshan .

M. P. Manohar

Department of Sugar Technology, Sir. M. Visvesvaraya Post

Graduate Centre, University of Mysore, Mandya 571 402,

Karnataka, India

e-mail: mahn_st2003@yahoo.com

\author{
Abbreviations \\ BHT Butylated hydroxytoluene \\ DPPH 1,1-Diphenyl-2-picrylhydrazyl \\ QDA Quantitative descriptive analysis \\ BHA Butylated hydroxyanisole \\ TBHQ tert-Butyl hydroquinone \\ TCA Trichloroacetic acid \\ BSA Bovine serum albumin \\ GAE Gallic acid equivalent \\ QE Quercetin equivalent \\ TAE Tannic acid equivalent \\ $\mathrm{EC}_{50}$ Effective concentration for $50 \%$ inhibition
}

\section{Introduction}

The control of oxidative processes during food processing and storage has an impact on the food quality. The oxidized food leads to off-flavors and decreases the organoleptic and nutritional quality of processed foods. Many synthetic antioxidants are in use in food industry to prevent oxidation in foods, but their usage is restricted for their toxicity issues (Botterweck et al. 2000). Plant and plant products serves as a source of natural antioxidants to improve the quality and nutritional value of foods. Among natural antioxidants, phenolic compounds are of special interest due to their wide distribution in the plant kingdom and potential antioxidant activity. Sugarcane contains various phenolic compounds and its extracts have displayed a wide range of biological activities including antioxidant, antiinflammatory, anti-thrombosis, immune-stimulation and anti-stress effects (El-Abasy et al. 2002; Molina et al. 2000; Ledon et al. 2003; Duarte-Almeida et al. 2006). 
Jaggery, a sugar rich plant product consumed worldwide is prepared traditionally by concentrating the juice of sugarcane (Saccharum officinarum). Jaggery has great nutritive and medicinal value. Indian Ayurvedic medicine considers jaggery as medicinal sugar for treating throat and lung infections. In vivo studies reported that a dietary supplement of jaggery was found to exhibit health benefits. Dietary jaggery reduced the development of atherosclerosis (Okabe et al. 2009), lowered the incidence of chromosomal aberration due to arsenic toxicity (Nrashanth et al. 2008) and exhibited protective effect against lung damage induced by coal, silica dust and other particulate matter (Sahu and Saxena 1994). Jaggery being a least processed sugar retains phenolics and other phytochemicals with potent biological activities like antioxidant, cytoprotective and anthelmintic activity as reported in literature (Harish Nayaka et al. 2009; Prasad et al. 2010).

Nutraceuticals are widely incorporated in functional foods owing to their therapeutic and health promoting effects. Jaggery enrichment with nutraceuticals may have an added health benefits. The use of and search for plant nutraceuticals for food enrichment have accelerated in recent years. Except the studies on jaggery fortification with vitamin $\mathrm{C}$-Indian gooseberry (Anwaar and Singh 2010) and sensory studies of jaggery chocolates (Khan Chand et al. 2011), the literature lacks information on jaggery enrichment with spices for their flavor and medicinal properties. Spices are esoteric food adjuncts used not only as a flavoring and coloring agents but also as folk medicine and food preservatives (Nakatani 1994; Cutler 1995). Ginger root (Zingiber officinale) is a rhizome belonging to the family Zingiberaceae, used medicinally and as a spice and food additive since antiquity for its characteristic flavor and pungency. Asian folk medicine uses ginger to treat a diverse array of ailments and has exhibited various pharmacological activities and as a food preservative (Shukla and Singh 2007; Nicoll and Henein 2009).

Therefore, studies on enrichment of jaggery with spices may lead to a better understanding of the effect of spice phytochemicals on various physico-chemical properties of jaggery. Hence, the present investigation is undertaken to evaluate physico-chemical properties, antioxidant activity and sensory evaluation of $Z$. officinale enriched jaggery from different sugarcane varieties.

\section{Materials and Methods}

Chemicals

1,1-Diphenyl-2-picrylhydrazyl (DPPH) purchased from Himedia Laboratories, Mumbai, India and Trolox from
Biomol Research Labs, Inc., USA. Coomassie Brilliant Blue G-250, Gallic acid, Trichloroacetic acid (TCA), Butylated hydroxytoluene (BHT) and Bovine serum albumin (BSA) procured from SISCO Research Laboratories Pvt. Ltd., Mumbai, India.

\section{Sugarcane Varieties}

Different varieties of sugarcane, viz. Co 86032, Co 419 and Co 62175 were procured from Zonal Agricultural Research Station, V. C. Farm, Mandya 571 405, Karnataka. All the sugarcane varieties obtained were cultivated in the same plot with similar management regimes.

\section{Zingiber officinale enriched jaggery preparation}

Zingiber officinale enriched jaggery was prepared following the method described by Jagannadha Rao et al. (2007). Z. officinale dried powder at different concentrations $(0.05$, 0.1 and $0.2 \%$ ) was added to sugarcane juice extracted from different sugarcane varieties and $\mathrm{pH}$ was adjusted to 6.6 using Milk of Lime $\left[\mathrm{Ca}(\mathrm{OH})_{2}\right]$. The juice was initially boiled for $10 \mathrm{~min}$ and the scum formed during boiling was completely removed through filtration using muslin cloth. Finally, the juice was heated and concentrated to thick syrup until the temperature reaches $118^{\circ} \mathrm{C}$. The scum formed after subsequent boiling was completely removed. The syrup was cooled and transferred to chocolate moulds to obtain desired shapes. Jaggery prepared without the addition of $Z$. officinale served as control. All the samples were stored at $4{ }^{\circ} \mathrm{C}$ in a sealed container for further analysis.

\section{Physico-chemical Characterization}

$p H$

$\mathrm{pH}$ was determined with a $\mathrm{pH}$ meter (Systronics India Pvt. Ltd., India) in a $5 \% \mathrm{w} / \mathrm{v}$ jaggery solution at room temperature (Guerra and Mujica 2010).

\section{Color}

The color of jaggery in $5 \% \mathrm{w} / \mathrm{v}$ solution was determined at $540 \mathrm{~nm}$ using visible spectrophotometer (Systronics India Ltd. Gujarat, India) with slight modification (Mandal et al. 2006).

\section{Turbidity}

The turbidity of jaggery was determined according to the method described by Guerra and Mujica (2010) with slight modification. Jaggery (5\%, w/v) was divided into two 
portions. One portion filtered through Whatmann No. 1 filter paper using siliceous earth as filtering aid was used as blank. The second portion was used to measure the percentage of transmittance at $720 \mathrm{~nm}$ using a visible spectrophotometer.

\section{Filterability}

Filterability of jaggery was measured following the method described by Guerra and Mujica (2010). The rate of filtration of $5 \%$ jaggery solution was compared to solution of pure sucrose, under the same conditions. Two hundred milliliters of pure sucrose $\left(28^{0}\right.$ Brix $)$ solution was filtered through Whatmann No. 1 filter paper and after $3 \mathrm{~min}$, the filtered volume measured and the procedure was repeated for jaggery samples. The percentage of filterability was calculated using the ratio of filtered volumes.

\section{Insoluble Solids}

Insoluble solids quantified as per the modified method described by Guerra and Mujica (2010). One gram of jaggery was dissolved in distilled water and filtered through Whatmann No. 1 filter paper. The residue on the filter paper was dried and weighed. The insoluble solids were expressed in percentage on dry weight basis.

\section{Water Activity $\left(a_{w}\right)$}

Water activity was determined using a water activity meter (Novasina, Switzerland) with technology based on the resistive electrolytic measurement principle.

Moisture, Protein, Ash, Reducing Sugars and Sucrose

Moisture, protein, ash, reducing sugars, sucrose content of jaggery was determined following the methods of Official AOAC (Helrich 1990). Results were expressed in percent on dry weight basis (except moisture).

\section{Determination of Total Phenol Content}

The total phenol content of jaggery was determined spectrophotometrically using Folin-Ciocalteu's method (Singleton et al. 1999). A sample aliquot of $100 \mu \mathrm{L}(5 \%)$ was added to $900 \mu \mathrm{L}$ of water, $1 \mathrm{~mL}$ of Folin-Ciocalteu reagent $(1: 2, \mathrm{v} / \mathrm{v})$ and $2 \mathrm{~mL}$ of $10 \%$ sodium carbonate sequentially, mixed thoroughly and incubated for one hour at room temperature. The absorbance was measured at $765 \mathrm{~nm}$ in visible spectrophotometer. Gallic acid was used as standard and the total phenolic content expressed as milligrams of Gallic acid equivalent (GAE) per gram sample.

\section{Determination of Total Flavonoid Content}

The total flavonoid content of jaggery was determined according to aluminium chloride method (Chang et al. 2002). A sample aliquot of $200 \mu \mathrm{L}(5 \%)$ was made up to $2 \mathrm{~mL}$ with distilled water. $100 \mu \mathrm{L}$ of $10 \%$ aluminium chloride and $100 \mu \mathrm{L}$ of $1 \mathrm{M}$ potassium acetate were added and incubated for $30 \mathrm{~min}$ at room temperature. The absorbance of the reaction mixture was read at $415 \mathrm{~nm}$. Quercetin was used as standard and total flavonoid content expressed in micrograms of quercetin equivalent per gram sample.

\section{Determination of Tannin Content}

The tannin content of jaggery was determined according to Folin-Ciocalteu's method (Singleton et al. 1999). A sample aliquot of $100 \mu \mathrm{L}(5 \%)$ was added to $900 \mu \mathrm{L}$ of water, $1 \mathrm{~mL}$ of Folin-Ciocalteu reagent $(1: 2, \mathrm{v} / \mathrm{v})$ and $2 \mathrm{~mL}$ of $10 \%$ sodium carbonate, mixed thoroughly and incubated for $1 \mathrm{~h}$ at room temperature. The absorbance was measured at $765 \mathrm{~nm}$ in visible spectrophotometer. Tannin acid was used as a standard and the total tannin content expressed as milligrams of tannic acid equivalent (TAE) per gram sample.

\section{Antioxidant Activity}

\section{DPPH Radical Scavenging Activity}

The DPPH radical scavenging activity of jaggery was evaluated as per the method described by Yamaguchi et al. (1998). An aliquot $(10-50 \mu \mathrm{L})$ of $10 \%$ jaggery samples and standard antioxidant (BHT) of various concentrations were made up to $200 \mu \mathrm{L}$ using distilled water and then mixed with $1 \mathrm{~mL}$ of $0.1 \mathrm{mM}$ DPPH in methanol. The mixture was shaken vigorously and allowed to stand for $20 \mathrm{~min}$ in the dark at room temperature. The absorbance of the resulting solution was read against control at $517 \mathrm{~nm}$ using a spectrophotometer. The ability to scavenge DPPH radical was calculated using the following equation.

DPPH Radical Scavenging ability $(\%)$

$=\left[\left(A_{\text {Control }}-A_{\text {sample }}\right) / A_{\text {Control }}\right] \times 100$

An effective concentration $\left(\mathrm{EC}_{50}\right)$ for $50 \% \mathrm{DPPH}$ radical scavenging activity was also calculated.

\section{Reducing Power Assay}

The reducing power of jaggery was determined according to the method reported earlier (Yen and Chen 1995). Different concentrations of jaggery $(1-5 \mathrm{mg} / \mathrm{mL})$ or standard antioxidant Trolox $(10-50 \mu \mathrm{g} / \mathrm{mL})$ was mixed with an 
equal volume of $0.2 \mathrm{M}$ Phosphate buffers, $\mathrm{pH} 6.6$ and $1 \%$ potassium ferricyanide. The mixture was incubated at $50{ }^{\circ} \mathrm{C}$ for $20 \mathrm{~min}$. An equal volume of $10 \%$ trichloroacetic acid was added to the mixture and centrifuged at $3,000 \mathrm{~g}$ for $10 \mathrm{~min}$. The upper layer of the solution was mixed with distilled water and $0.1 \% \mathrm{FeCl}_{3}$ at a ratio of 1:1:2(v/v/v) and the absorbance measured at $700 \mathrm{~nm}$. The increased absorbance of the reaction mixture indicated increased reducing power.

\section{Sensory Attributes}

Zingiber officinale enriched jaggery were evaluated for sensory attributes like color, texture, hardness, chewiness, sweetness, saltiness, pleasantness, spicy and overall acceptance by unstructured scaling method or quantitative descriptive analysis (QDA) (Stone et al. 1974). The panel consisted of 20 people in the group of 20-50 years comprising both male and female, often participated in sensory evaluation of jaggery samples. The descriptors of sensory attributes explained orally to the panelists. Information on $Z$. officinale enriched jaggery was given to a panelist; however, they lacked knowledge of varying percent and type of cane variety. The scorecard consisted of a horizontal line $15 \mathrm{~cm}$ long with anchor points $1.5 \mathrm{~cm}$ from each end. Each anchor point labeled with a word or expression. A separate horizontal line used for each sensory attribute evaluated. Panelists asked to record each evaluation, by marking a vertical line across the horizontal line at the point according to their intensity or perception of the magnitude of each attribute.

Coded jaggery samples in individual re-sealable bags were served to panelist one at a time and the presentations of the samples randomized. Panelists provided with water and asked to rinse their mouth after evaluating each sample. After the panelist judgment, the distance from the left end of the line to each point marked by the panelist measured and the distance measured recorded as intensity rating between 0.0 and 15.0 for each product evaluated and analyzed statistically.

\section{Statistical Analysis}

All the experiments were carried out in triplicates $(n=3)$ and the results expressed as mean \pm standard deviation (SD) using Microsoft Excel software. The sensory scores subjected to analysis of variances (ANOVA) to determine statistically significant $(P \leq 0.05)$ preferences in sensory attributes. Post hoc comparisons made by least significant difference (LSD) and Duncan's multiple range test (DMRT). IBM SPSS Statistical software version 19.0 used to analyze the results.

\section{Results and Discussion}

Physico-chemical Characterization of Z. officinale Enriched Jaggery

The results of physical properties like $\mathrm{pH}$, color, moisture, turbidity, filterability, insoluble solids, water activity and ash content of $Z$. officinale enriched jaggery are represented in Table 1.

The $\mathrm{pH}$ of $Z$. officinale enriched jaggery and its controls of all sugarcane varieties were in the range of 5.66-5.87 that supports the results reported by Guerra and Mujica (2010).The observed $\mathrm{pH}$ of jaggery was marginally lower than 5.9 as prescribed by Ecuadorian technical standard (2002) for panela. Lowered $\mathrm{pH}$ of jaggery may be due to deficiency of lime added for juice clarification.

Based on the absorbance at $540 \mathrm{~nm}$, the color intensity of jaggery was appraised. Color of jaggery finds to be the primary factor for consumer preference and market, and is dependent on dark compounds formed during processing. Jaggery browning occurs due to caramelization of sugars, oxidation of phenolic compounds, alkaline decomposition of sucrose or by Maillard reaction (Damodaran 2000). Z. officinale enrichment jaggery of all sugarcane varieties showed elevated absorbance with increased concentration of $Z$. officinale compared to its respective controls. Control jaggery $(0 \%)$ of Co 86032 variety had least color (golden brown) among all controls and enriched jaggery. Hence, darkened color was resulted in all sugarcane variety jaggery upon enrichment with $Z$. officinale.

Moisture content and water activity are two important parameters determine the quality, stability and shelf-life of foods during storage. $Z$. officinale enrichment showed a marked increase $(1 \%)$ in moisture content at $0.05 \%$ concentration for Co 86032 and Co 62175 jaggery but a very slight increase in moisture content observed for $\mathrm{Co}$ 419 jaggery at the same concentration. In addition, moisture content of jaggery further increased with increase $Z$. officinale concentrations in all sugarcane varieties. Variation in moisture content within the varieties occurs during jaggery processing at the final stages. Water activity, $\mathrm{a}_{\mathrm{w}}$ represents the water status in the food system and governs microbial growth (Beuchat 1987; Troller and Christian 1978). $a_{w}$ of $Z$. officinale enriched jaggery was varied between six and 7 and the results were similar to the results reported for panela (Guerra and Mujica 2010). However, marginally synergism lowering of $\mathrm{a}_{\mathrm{w}}$ observed in Z. officinale enriched jaggery of all three sugarcane varieties. These results indicated that $Z$. officinale enrichment could offer better shelf-life and promising quality for jaggery during storage. However, $\mathrm{a}_{\mathrm{w}}$ in the range $0.60-0.65$ finds to be the optimum condition for growth osmophilic and xerophilic microbes such as Aspergillus euinulatus, 


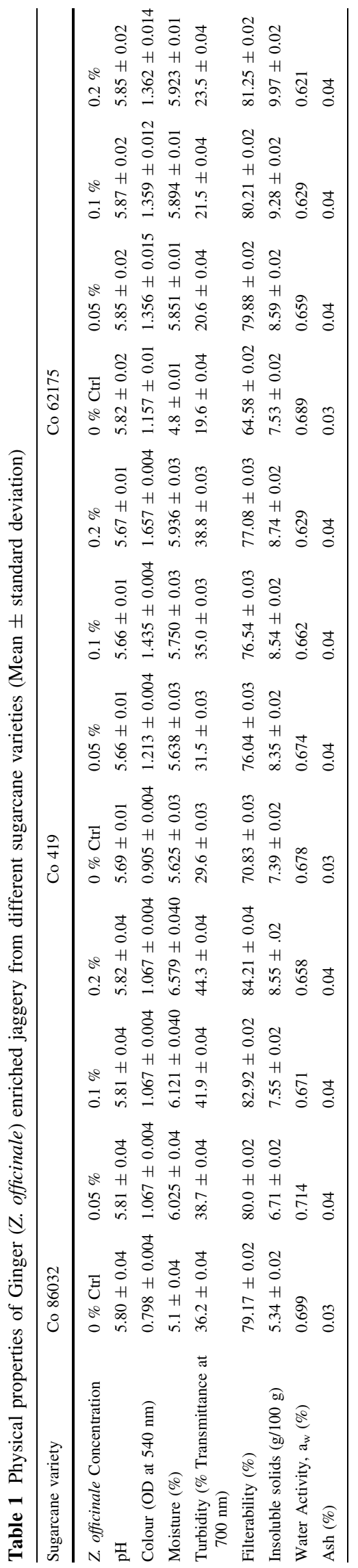

Aspergillus candidus, Aspergillus chevalieri, Saccharomyces rouxiiand Saccharomyces bisporus and thus supports their growth on jaggery and results in spoilage (Beuchat 1981).

Turbidity of $Z$. officinale enriched jaggery quantified by measuring percentage transmittance at $720 \mathrm{~nm}$. Z. officinale enriched jaggery of all sugarcane varieties showed a gradual increase in turbidity from its control with dose dependent enrichment. Both control and enriched jaggery of Co 62175 variety found to show lower turbidity than Co 86032 and Co 419 variety's jaggery. About 8-9 \%, increase in turbidity was observed between control and enriched jaggery at $0.2 \% \mathrm{Z}$. officinale concentration in both Co 86032 and Co 419 variety's jaggery. Increased turbidity of $Z$. officinale enriched jaggery demonstrated that $Z$. officinale could be the last option for juice clarification.

Zingiber officinale enriched jaggery showed a marginal increase in filterability upon spice enrichment with its control of Co 86032 variety. However, results showed initial remarkable increased (6 and $15 \%$ ) filterability at $0.05 \%$ Z. officinale enriched jaggery of the varieties Co 419 and Co 62175 respectively than its control and slightly progressive increased filterability with increase in $Z$. officinale enrichment. In addition, $Z$. officinale enriched jaggery showed continuous raise in insoluble solid contents with dose enhanced spice enrichment but the ash content was differed by $0.01 \%$ in enriched jaggery of all sugarcane varieties irrespective of their $Z$. officinale concentrations from its control.

The results of chemical properties like sucrose, reducing sugars, proteins, total phenols, tannins and total flavonoids of $Z$. officinale enriched jaggery are represented in Table 2 .

Sucrose and reducing sugar content of $Z$. officinale enriched jaggery of different sugarcane varieties showed no significant difference upon Z. officinale addition from its control. However, a very marginal increase in both sucrose and reducing content observed for all sugarcane variety's jaggery upon $Z$. officinale enrichment and followed dose dependent spice addition.

Protein content of $Z$. officinale enriched jaggery as determined by Bradford's method indicated no significant difference in protein content between enriched and control of Co 86032 and Co 419 variety's jaggery. Z. officinale enrichment showed a synergistic increase in protein content for Co 62175 jaggery and about $0.4 \mathrm{mg} / \mathrm{g}$ of protein content enhanced at $0.2 \% \mathrm{Z}$. officinale addition than its control.

A dose dependency increase in total phenol, tannin and flavonoid contents was resulted in Z. officinale enriched jaggery in all sugarcane varieties. Jaggery prepared from Co 86032, Co 419 and Co 62175 exhibited increase in 11.1, 12.0 and $16.5 \%$ phenol; $15.4,14.6$ and $16.2 \%$ tannin and 10.6, 6.7 and $7.7 \%$ flavonoid contents, respectively from its control at $0.05 \% \mathrm{Z}$. officinale enrichment. 


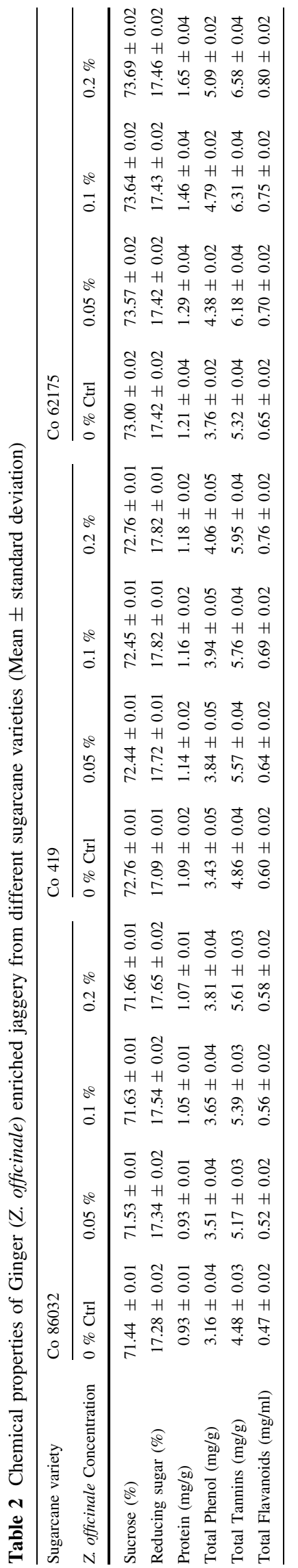

Antioxidant Activity of Z. officinale Enriched Jaggery

Antioxidant activity of $Z$. officinale enriched jaggery was measured by two in vitro assays, i.e., DPPH radical scavenging ability and reducing power assay. The antioxidant activity of plant extracts containing polyphenols is due to their ability to be donors of hydrogen atoms or electrons and to capture the free radicals. DPPH assay is one of the tests used to prove the ability of the components of the $Z$. officinale enriched jaggery to act as donors of hydrogen atoms. DPPH is a stable free radical, and in its radical form absorbs at $517 \mathrm{~nm}$ whose absorption decreases after acceptance of an electron or hydrogen atom from an antioxidant due to the formation of its non-radical form DPPH-H (Blios 1958). The degree of decolorization of DPPH is a stoichiometric measure of the antioxidant potential of test samples. The scavenging ability of $Z$. officinale enriched jaggery of all three sugarcane varieties were expressed in terms of $\mathrm{EC}_{50}$ values as shown in Table 3. All enriched jaggery showed concentration dependent free radical scavenging activity. Z. officinale enrichment decreased $\mathrm{EC}_{50}$ concentration than its control jaggery irrespective of sugarcane varieties. At $0.2 \%$ enrichment, Jaggery of Co 86032, Co 419 and Co 62175 had $\mathrm{EC}_{50}$ of $3.098,3.076$ and $3.038 \mathrm{mg} / \mathrm{mL}$, respectively. $\mathrm{EC}_{50}$ of BHT, used as standard was $7.5 \mu \mathrm{g} / \mathrm{mL}$. Both enriched and control jaggery showed higher (450 folds) $\mathrm{EC}_{50}$ concentration than standard BHT. Results of DPPH radical assay showed a positive correlation $(\mathrm{r}=0.922$, 0.883 and 0.881 ) with total phenolics of Co 86032, Co 419 and Co 62175 jaggery, respectively. High correlations between total phenolics and scavenging of DPPH radical indicated that polyphenols present in the $Z$. officinale enriched jaggery are the main antioxidants.

Further, reducing capacity assay provides a measure of compound's ability to donate electrons and reduce the oxidized intermediates formed in peroxidation process. The assay is based on the reduction of $\mathrm{Fe}^{+3}$-ferricyanide complex that is monitored by measuring the formation of perl's blue at $700 \mathrm{~nm}$. Increasing absorbance indicates an increase in reductive ability (Olayinka and Anthony 2010). Since reducing power of a compound serves as a significant indicator of its antioxidant activity (Meir et al. 1995), Z. officinale enriched jaggery assayed for reducing power ability. In Fig. 1, Z. officinale enriched jaggery of all cane varieties exhibited in vitro ferric reducing potential in a dose dependent manner. The absorbance of enriched jaggery at $700 \mathrm{~nm}$ had increased with increase in spice enrichment than its respective controls. Trolox, used as a standard showed absorbance of 1.39 at $50 \mu \mathrm{g} / \mathrm{mL}$. The reducing potential of $Z$. officinale enrich jaggery $(0.2 \%)$ increased by 23.22, 26.00 and $24.53 \%$ than its control of Co 86032, Co 419 and Co 62175, respectively. 


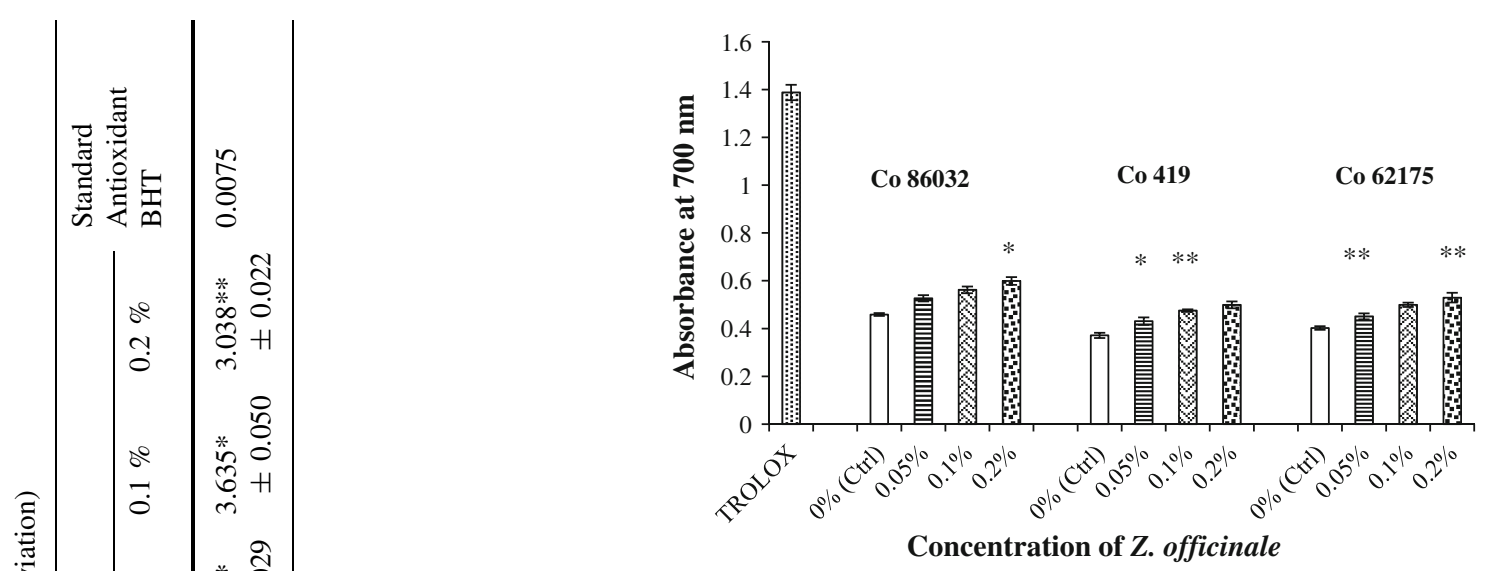

Fig. 1 Reducing power of Ginger (Z. officinale) enriched jaggery $(5 \mathrm{mg} / \mathrm{mL})$ from different sugarcane varieties and standard Trolox antioxidant $(50 \mu \mathrm{g} / \mathrm{mL})(* P<0.05 ; * * P<0.01, * * * P<0.001)$

Natural antioxidants play an important role in the prevention and interception of oxidative damage and have great impact on the safety and acceptability of the food system. They keep the food stable against oxidation and act as a potent preservative by controlling microbial growth. The traditional practice of adding antioxidants during processing can still play a very important role as added compounds have additional potential for enhancing endogenous antioxidant systems. In addition, antioxidant activity of plant is often associated with polyphenols that with hydrogen donating capacity inhibits free radical induced oxidation (Yen et al. 1993). The phenolic compounds of sugarcane juice exhibited antioxidant potential (Duarte Almeida et al. 2006) and conferred various biological activities. The antioxidant compounds extracted from jaggery showed stronger antioxidant potential than BHT in earlier reports (Nakasone et al. 1996). In our own studies (Harish Nayaka et al. 2009) jaggery showed strong DPPH radical scavenging ability $\left(\mathrm{EC}_{50}-7.81 \mu \mathrm{g} / \mathrm{mL}\right)$ and reducing capacity with absorbance of 2.66 at $20 \mathrm{mg} /$ $\mathrm{mL}$ at $700 \mathrm{~nm}$. A plant-derived food additive, especially polyphenolic compounds has been ascribed health-promoting properties, as for example in terms of prevention of chronic cardiovascular diseases (Harborne and Williams 2000). Z. officinale rhizome used as spices and condiments in many food preparations found to possess both in vitro and in vivo antioxidant action and protection against free radical damage (Masuda et al. 2004; Ahmed et al. 2000). In addition, active ingredients of rhizome also contributed for a wide array of biological activities (Young et al. 2005). In present investigation, Z. officinale enrichment during jaggery processing resulted synergistic increase in both total phenolic content and anti-oxidative potential of jaggery, and hence the combination of nutritional and medicinal benefits determines ginger-jaggery as a functional food. 
A

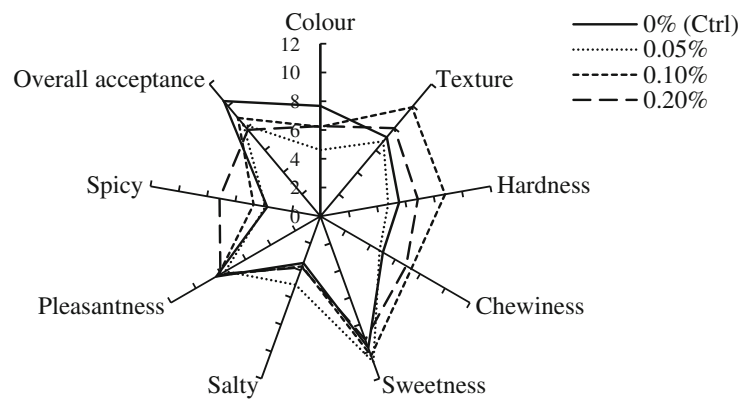

Co 86032

B

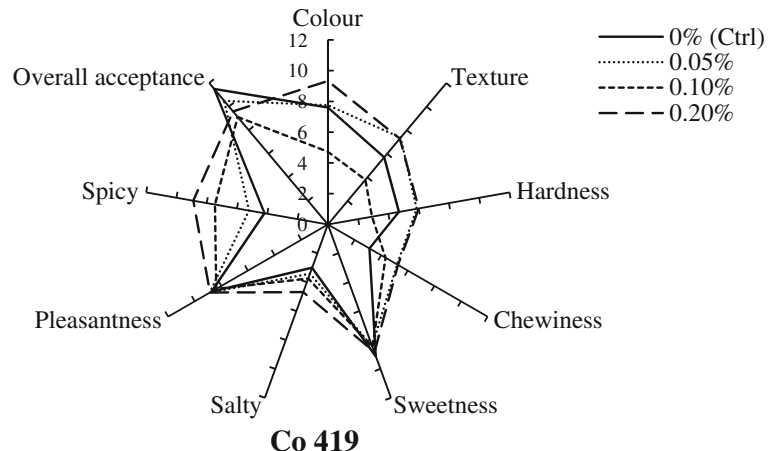

C

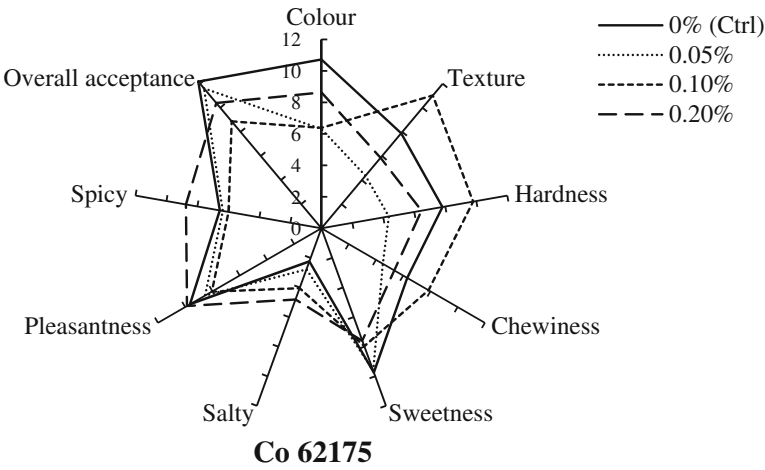

Fig. 2 Star diagram of sensory attributes of Ginger (Z. officinale) enriched jaggery from Co 86032 (a), Co 419 (b) and Co 62175 (c) sugarcane varieties

\section{Sensory Attributes of Z. officinale Enriched Jaggery}

Sensory attributes such as color, texture, hardness, chewiness, sweetness, salty, pleasantness, spicy and overall acceptance of $Z$. officinale enriched jaggery of three sugarcane varieties were evaluated by a quantitative descriptive analysis method (Fig. 2). Z. officinale enriched jaggery of Co 86032, Co 419 and Co 62175 had showed a statistical significant difference for sensory attributes such as color, texture, hardness, chewiness and spicy. However, data indicated texture, hardness, chewiness and spicy attributes enhanced in dose dependent $Z$. officinale enrichment. Neither sweetness nor pleasantness altered upon spice enrichment but panelist preferred much acceptance for control jaggery than $Z$. officinale enriched jaggery.

\section{Conclusions}

The results of the present investigation revealed that the addition of $Z$. officinale dried powder during jaggery preparation from different sugarcane varieties indicated an enhanced phenolic content and antioxidant potential as evidenced by DPPH radical scavenging and reducing power assays. The sucrose and reducing sugar content of $Z$. officinale enriched jaggery not affected upon enrichment of $Z$. officinale. The sweetness and pleasantness of jaggery were same as that of the control for all sugarcane varieties tested. Hence, Z. officinale enriched jaggery used as a substitute for regular jaggery with additional health benefits.

Acknowledgments The authors wish to thank Dr. Swamy Gowda, Sugarcane Breeder, Zonal Agricultural Research Station, V.C. Farm, Mandya, Karnataka, India for providing sugarcanes of different varieties for our study. Miss C. Vinutha acknowledges the Department of Science and Technology, New Delhi, India for awarding the INSPIRE fellowship.

\section{References}

Ahmed, R.S., V. Seth, S.T. Pasha, and B.D. Banerjee. 2000. Influence of dietary ginger (Zingiber officinales Rosc.) on oxidative stress induced by malathion in rats. Food and Chemical Toxicology 38: 443-450.

Anwaar, S. I., and J. Singh. 2010. Modified pans for open pan jaggery furnace. Operation Manual No. AE/10/01, Lucknow: Indian Institute of Sugarcane Research.

Beuchat, L.R. 1987. Influence of water activity on growth, metabolic activities and survival of yeasts and molds. Journal of Food Protection 46: 135-141.

Beuchat, L.R. 1981. Microbial stability as affected by water activity. Cereal Foods World 26: 345-349.

Blios, M.S. 1958. Antioxidant determinations by the use of a stable free radical. Nature 181: 1199-1200.

Botterweck, A.A.M., H. Verhagen, R.A. Goldbohm, J. Kelinjans, and P.A.V.D. Brandt. 2000. Intake of butylatedhydroxyanisole and butylatedhydroxytoluene and stomach cancer risk: Results from analyses in the Netherlands cohort study. Food and Chemical Toxicology 38: 599-605.

Chang, C., M. Yang, H. Wen, and J. Chern. 2002. Estimation of total flavonoid content in propolis by two complementary colorimetric methods. Journal of Food and Drug Analysis 10: 178-182.

Cutler, H.G. 1995. Natural product flavor compounds as potential antimicrobials, insecticides, and medicinals. Agro-Food-Industry Hi-Tech 6: 19-23.

Damodaran, S. 2000. Aminoacidos, peptidos y proteinas. In: Fenemma, O. (ed.). Quimica de alimentos. 2 edn. Espana: Acribia.

Duarte-Almeida, J.M., A.V. Novoa, A.F. Linares, F.M. Lajolo, and M.I. Genovese. 2006. Antioxidant activity of phenolics compounds from sugarcane (Saccharum officinarum L.) juice. Plant Foods for Human Nutrition 61: 187-192. 
Ecuadorian Technical Standard NTE INEN 2 332. 2002. Panela Granulada Requisitos (p. 2) Quito-Ecuador.

El-Abasy, M., M. Motobu, K. Shimura, K.J. Na, C.B. Kang, K. Koge, T. Onodera, and Y. Hirota. 2002. Immunostimulating and growth promoting effects of sugarcane extracts (SCE) in chickens. Journal of Veterinary Medical Science 64: 1061-1063.

Guerra, M.J., and M.V. Mujica. 2010. Physical and chemical properties of granulated cane sugar "panelas". Cienciae Tecnologia de Alimentos, Campinas 30: 250-257.

Harborne, J., and C. Williams. 2000. Advances in flavonoid research since 1992. Phytochemistry 55: 481-504.

Harish Nayaka, M.A., U.V. Sathisha, M.P. Manohar, K.B. Chandrashekar, and M.D. Shylaja. 2009. Cytoprotective and antioxidant activity studies of jaggery sugar. Food Chemistry 115: 113-118.

Jagannadha Rao, P.V.K., D. Madhusweta, and S.K. Das. 2007. Jaggery-A Traditional Indian Sweetener. Indian Journal of Traditional Knowledge 6: 95-102.

Helrich, Kenneth. 1990. Official methods of analysis of the Association of Official Analytical Chemists. 15th eds, 777-781. Washington DC.

Chand, Khan, Anupama Singh, and A.K. Verma. 2011. Quality evaluation of jaggery chocolates under various storage conditions. Sugar Tech 13: 150-155.

Ledon, N., A. Casaca, V. Rodriguez, J. Cruz, R. Gonzalez, Z. Tolon, M. Cano, and E. Rojas. 2003. Antiinflammatory and analgesic effects of a mixture of fatty acids isolated and purified from sugarcane wax oil. Planta Medica 69: 367-369.

Mandal, D., S.R. Tudu, Mitra, and G.C. De. 2006. Effect of common packing material on keeping quality of sugarcane jaggery during monsoon season. Sugar Tech 8: 2-3.

Masuda, Y., H. Kikuzaki, M. Hisamoto, and N. Nakatani. 2004. Antioxidant properties of gingerol related compounds from ginger. BioFactors 21: 293-296.

Meir, S., J. Kanner, B. Akiri, and S.P. Hadas. 1995. Determination and involvement of aqueous reducing compounds in oxidative defense systems of various senescing leaves. Journal of Agricultural and Food Chemistry 43: 1813-1817.

Molina, V., M.L. Arruzazabala, D. Carbajal, R. Mas, and S. Valses. 2000. Antiplatelet and antithrombotic effect of D-003. Pharmacological Research 42: 137-143.

Nakatani, N. 1994. Antioxidative and antimicrobial constituents of herbs and spices. In Spices, herbs and edible fungi, ed. G. Charalambous, 251-271. New York: Elsevier Science.

Nicoll, R., and M.Y. Henein. 2009. Ginger (Zingiber officinale Roscoe): A hot remedy for cardiovascular disease? International Journal of Cardiology 131: 408-409.

Singh, Nrashant, D. Kumar, S. Raisuddin, and P.S. Anand. 2008. Genotoxic effects of arsenic: Prevention by functional foodJaggery. Cancer Letters 268: 325-330.
Okabe, T.T. Toda, M. Inafuku, K. Wada, H. Iwasaki, and H. Oku. 2009. Antiatherosclerotic functions of kokuto, Okinawan noncentrifuged cane sugar. Journal of Agricultural and Food Chemistry 57: 69-75.

Olayinka, A.A., and I.O. Anthony. 2010. Preliminary phytochemical screening and In vitro antioxidant activities of the aqueous extract of Helichrysum longifolium DC. BMC Complementary and Alternative Medicine 10: 21-25.

Prasad, P., P. Praveen Kumar, K. Satyavathi, and M.C. Prabhakar. 2010. Comparatives studies on anthelmintic activity of natural sweeteners. Research Journal of Pharmaceutical, Biological and Chemical Sciences 1: 510-514.

Sahu, A.P., and A.K. Saxena. 1994. Enhanced translocation of particles from lungs by jaggery. Environmental Health Perspectives 102: 211-214.

Shukla, Y., and M. Singh. 2007. Cancer preventive properties of ginger: A brief review. Food Chemistry and Toxicology 45: 683690.

Singleton, V.L., R. Orthofer, and R.M. Lamuela-Raventos. 1999. Analysis of total phenols and other oxidation substrates and antioxidants by means of Folin-Ciocalteu reagent. Methods in Enzymology 299: 152-178.

Stone, H., J. Sidel, S. Oliver, A. Woolsey, and R.C. Singleton. 1974. Sensory evaluation by quantitative descriptive analysis. Food Technology 28: 24-34.

Troller, J.A., and J.H.B. Christian. 1978. Water activity and food. New York: Academic Press.

Yamaguchi, T., H. Takamura, T. Matoba, and J. Terao. 1998. HPLC method for evaluation of the free radical-scavenging activity of foods by using 1,1-diphenyl-2-picrylhydrazyl. Bioscience, Biotechnology, and Biochemistry 62: 1201-1204.

Yen, G.C., and H.Y. Chen. 1995. Antioxidant activity of various tea extracts in relation to their antimutagenicity. Journal of Agricultural and Food Chemistry 43: 27-32.

Yen, G.C., P.D. Duh, and C.L. Tsai. 1993. The relationship between antioxidant activity and maturity of peanut hulls. Journal of Agricultural and Food Chemistry 41: 67-70.

Nakasone, Yoko, Kensaku Takara, Kouji Wada, Junichi Tanaka, Seiichi Yogi, and Nobuji Nakatani. 1996. Antioxidative compounds isolated from Kokuto, Non-centrifugal cane sugar. Bioscience, Biotechnology, and Biochemistry 60: 1714-1716.

Young, H.Y., Y.L. Luo, H.Y. Cheng, W.C. Hsieh, J.C. Liao, and W.H. Peng. 2005. Analgesic and anti-inflammatory activities of [6]-gingerol. Journal of Ethnopharmacology 96: 207-210. 